\title{
Entzündliche Herzmuskelerkrankung als Ursache einer Herzinsuffizienz
}

\author{
Felicitas Escher, Carsten Tschöpe, Heinz-Peter Schultheiss
}

\begin{tabular}{|c|c|c|c|}
\hline \multicolumn{4}{|l|}{ Übersicht } \\
\hline Einleitung & 25 & Pathophysiologische Aspekte der & \\
\hline Charakteristika der entzündlichen & & Myokarditis & 27 \\
\hline Herzerkrankungen & 25 & Klinisches Bild und Diagnostik & 29 \\
\hline Ätiologie & 26 & Epidemiologie und Prognose & 32 \\
\hline & & Therapeutische Implikationen & 33 \\
\hline
\end{tabular}

\section{Einleitung}

Kardiomyopathien sind eine wesentliche Ursache für die Entwicklung einer Herzinsuffizienz. Dabei ist die entzündliche Kardiomyopathie eine eigene Krankheitsentität, deren Pathomechanismen in den letzten Jahren weiter aufgeklärt wurden. Ihre Symptome sind vielfältig - genauso wie auch das Spektrum der möglichen Ursachen, wobei die Ätiologie der Erkrankung prognostisch relevant ist $[1,2]$. Dennoch wird heute oft nur symptomatisch therapiert, obwohl histologische, immunhistochemische und molekularbiologische Untersuchungsverfahren zur Klärung der Ursache zur Verfügung stehen $[4,5,6]$. Mit diesen Verfahren können die Patienten identifiziert werden, die von einer spezifischen Therapie profitieren. Ziel dieser Übersicht ist es, die vorhandene Evidenz zu Ätiologie, Pathophysiologie und Therapie darzustellen.

\section{Charakteristika der entzündlichen Herzerkrankungen}

Eine Peri-Myokarditis ist klinisch und pathologisch als Entzündungsprozesse des Peri- oder Myokardgewebes definiert.

\section{Epidemiologie}

Weil die Beschwerden sehr verschieden sein können, ist die Inzidenz der Myokarditis nicht sicher bekannt, wird aber bei Autopsien in 1-9\% gefunden. Die Myokarditis ist vor allem bei jungen Patienten eine häufige Ursache einer Herzinsuffizienz. Die Prävalenz der histologisch nachweisbaren Entzündung in Myokardbiopsien von Patienten mit akut aufgetretener Herzinsuffizienz beträgt 10-40\% [7]. Bei Patienten mit dem klinischen Vollbild einer dilatativen Kardiomyopathie konnte sogar in $60 \%$ eine (virusassoziierte) chronische Entzündung nachgewiesen werden.

\footnotetext{
Auch bei systemischen Erkrankungen wird eine Herzbeteiligung in $10-20 \%$ angenommen.
}

\section{Definition und Einteilung}

An den bereits bestehenden Definitionen der Myokarditis durch die Dallas- [5] und die WHO/ISCF-Kriterien von 1996 [8] wurde in dem Konsensus-Statement der „European Society of Cardiology (ESC) Working Group on Myocardial and Pericardial Diseases" festgehalten: Die Myokarditis ist histologisch durch den Nachweis entzündlicher Infiltrate im Myokard, assoziiert mit Myozytendegeneration und Nekrose einer nicht ischämischen Ursache definiert. Die Kriterien wurden dabei noch präzisiert durch den quantitativen Nachweis von 
mindestens 14 Leukozyten $/ \mathrm{mm}^{2}$ im Myokard (inkl. $\leq 4$ Monozyten $/ \mathrm{mm}^{2}$ ) mit dem Nachweis von 7 oder mehr CD3-positiven T-Lymphozyten [9].

Zudem wurde die folgende Unterteilung der Myokarditis angeregt:

- die Virus-Myokarditis mit histologischem/immunhistochemischem Nachweis intramyokardialer Inflammation mit Nachweis kardiotroper Erreger

- die Autoimmun-Myokarditis mit histologischem/ immunhistochemischem Nachweis einer Myokarditis, allerdings ohne Nachweis kardiotroper Erreger (mit oder ohne kardiale Autoantikörper)

\section{Ätiologie}

\section{Erregerspektrum}

Obwohl letztlich jeder infektiöse Erreger eine myokardiale Inflammation auslösen und in der Folge zu einer linksventrikulären Dysfunktion führen kann, ist die Virus-Myokarditis die häufigste entzündliche Herzmuskelerkrankung. Durch den routinemäßigen Einsatz molekularbiologischer Methoden hat sich das Spektrum der kardiotropen Viren erheblich erweitert, sodass die folgenden Viren als epidemiologisch relevant gelten müssen: Enteroviren (EV), Adenoviren (ADV), Parvovirus B19 (B19V), Humanes-Herpesvirus Typ 6 (HHV 6), Epstein-Barr-Virus (EBV) sowie Zytomegalievirus (hier insbesondere bei immunsupprimierten Patienten). In ca. 30\% liegen Mehrfachinfektionen des Myokards vor [10].

Bei anderen viralen Infektionen (z. B. Influenzaviren, Hepatitis-C-Virus, HIV) ist die ätiologische Bedeutung im Sinne einer direkten Virusvermehrung im menschlichen Herz nicht gesichert, wobei klinische und bioptische Arbeiten belegen, dass sich eine Myokarditis auch im Rahmen systemischer Immunprozesse ohne direkte Virusvermehrung im Herzmuskel manifestieren kann.

\section{Häufige Erreger}

In geografisch unterschiedlichen Häufigkeiten wurden früher vor allem EV (Coxsackieviren der Gruppe A, verschiedenen Coxsackieviren der Gruppe B sowie Echoviren) und ADV molekularbiologisch nachgewiesen. Humanpathogene EV, insbesondere Coxsackieviren der Gruppe B, replizierten dabei vorzugsweise in Myozyten und konnten in allen Stadien einer akuten und chronischen enterovirusinduzierten Myokarditis nachgewiesen werden [11]. In der Studie von Bowles et al. wurden bei $40 \%$ von 773 Amerikanern unter 18 Jahren, bei denen der klinische Verdacht auf eine Myokarditis $(n=624)$ oder auf eine dilatative Kardiomyopathie $(n=149)$ bestand, vor allem Coxsackieviren und Adenoviren mittels Nested-PCR nachgewiesen [12].

In der letzten Dekade wandelte sch das Erregerspektrum hin zu Erythroviren, inkl. B19V (bis 45\%), EBV und HHV 6, während die „klassischen“ Myokarditiserreger seltener nachgewiesen wurden [13].

Erregerspektrum im Myokard: heute insbesondere B19V, EBV und HHV 6.

\section{Andere Erreger}

Myokarditiden bei bakteriellen oder parasitären Infektionen, wie z.B. die Chagas-Erkrankung in Südamerika, haben in Endemieregionen eine große Bedeutung, sind aber in westlichen Industrieländern sehr selten. Die bei Borreliosen in ca. 10\% angenommene kardiale Beteiligung manifestiert sich überwiegend während der ersten 3 Monate nach Infektion mit meist reversiblen Reizleitungsstörungen, wobei das histologische Bild häufig einer lymphozytären Virusmyokarditis gleicht, aber auch ohne nachweisbare Entzündungsprozesse auftreten kann. Der Erreger kann trotz antibiotischer Therapie im Herz persistieren.

Rückläufig ist die rheumatische Myokarditis als allergisch-hyperergische Reaktion mit Ausbildung rheumatischer Granulome im Rahmen des durch $\beta$-hämolysierende Streptokokken ausgelösten rheumatischen Fiebers.

\section{Nicht infektiöse Ursachen}

Auch eine Reihe nicht infektiöser Ursachen kommt für eine Myokarditis infrage, wobei neben entzündlichen Systemerkrankungen (z. B. Sarkoidose, rheumatoide Arthritis, Lupus erythematodes, Sklerodermie, Dermatomyositis) auch eine Arzneimittelüberempfindlichkeit (z.B. Antibiotika, Clozapin, Mesalazin) und die Kardiotoxizität von z.B. Anthrazyklinen oder Kokain klinisch wichtig sind.

Eine Sonderrolle nehmen die selten vorkommende idiopathische Riesenzellmyokarditis und die eosinophile Myokarditis ein, da sie fulminant - und unbehan- 
delt oft tödlich - verlaufen. Charakteristisch für die Riesenzellmyokarditis ist der histologische Befund mit massiver Destruktion der Myokardzellen durch mehrkernige Riesenzellen, Lymphozyten, Plasmazellen und eosinophilen Granulozyten. Die nekrotisierende eosinophile Myokarditis manifestiert sich häufig als Hypersensitivitätsmyokarditis und seltener als hypereosinophiles Syndrom.

\section{Pathophysiologische Aspekte der Myokarditis}

\section{Virale Myokarditis}

\section{Verlauf}

Die virale Myokarditis verläuft phasenweise (Abb. 1):

- Zunächst wird das Virus im Rahmen eines generellen Infekts rezeptorvermittelt in die Myokardzelle aufgenommen und repliziert sich dort. Noch bevor die Immunantwort einsetzt, kann das Virus das Myokard schädigen.
- Die Stimulation einer adäquaten zellulären und humoralen Immunantwort führt häufig zur Lyse virusbefallener Herzmuskelzellen mit konsekutiver Viruselimination und Ausheilen der Entzündung. In Abhängigkeit von bislang letztlich noch nicht vollständig bekannten Faktoren kann sich das Virus jedoch bei einem Teil der Patienten der Überwachung durch das Immunsystem entziehen, wodurch eine persistierende Infektion induziert werden kann, die mit einer chronischen Entzündungsreaktion des Herzmuskels einhergeht.

- Die letzte Phase kann inflammatorische und postinflammatorische Anteile in der Endomyokardbiopsie (EMB) aufweisen. Vom akuten Koronarsyndrom bis zu supraventrikulären und ventrikulären Herzrhythmusstörungen mit plötzlichem Herztod, vom „Chronic-fatigue-Syndrom“ und leichter LV-Dysfunktion bis zum Vollbild der dilatativen Kardiomyopathie sind alle Erscheinungsformen möglich.

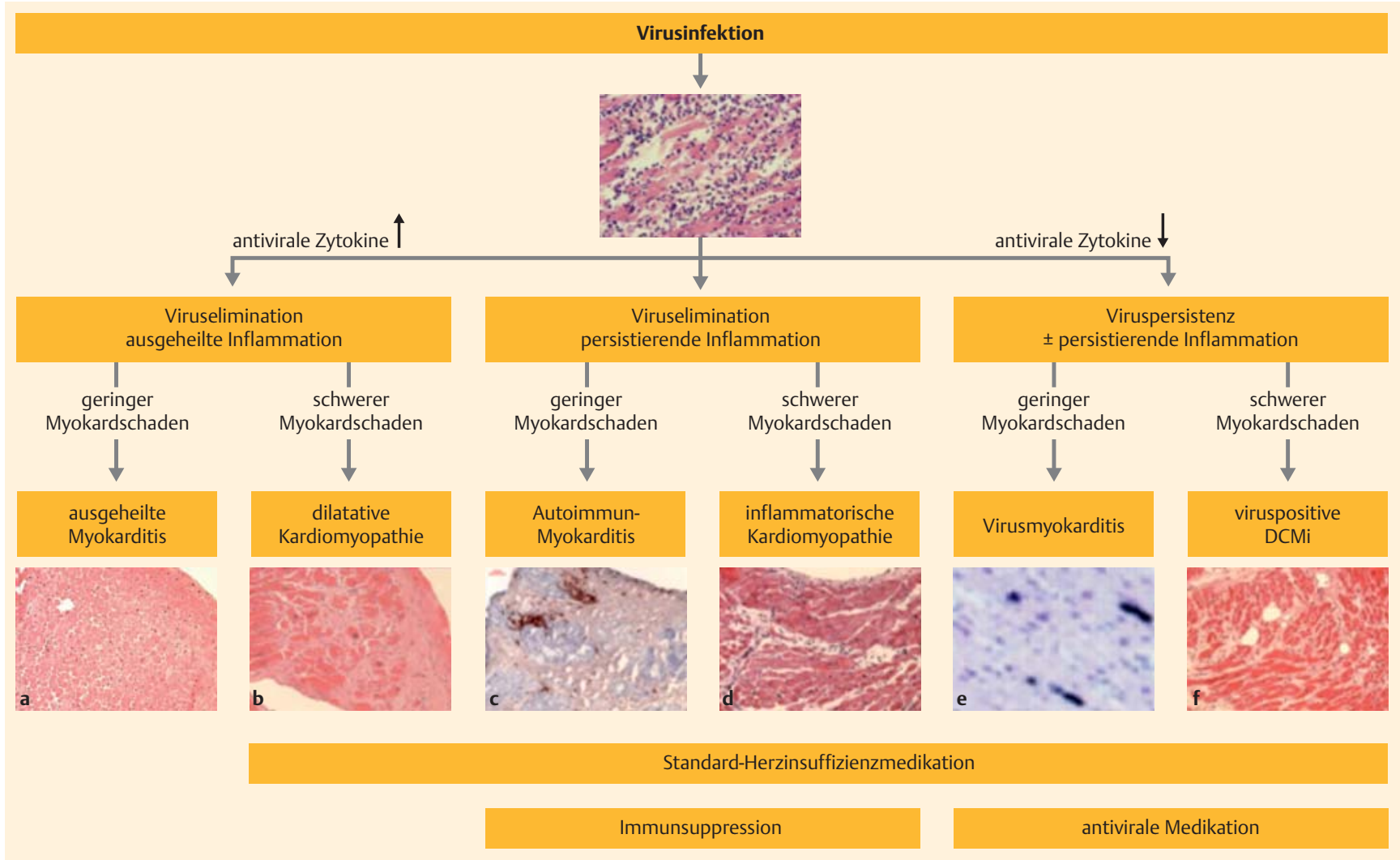

Abb. 1 Pathogenese der Virusmyokarditis und inflammatorischen Kardiomyopathie modifiziert nach [4]. a HE-Färbung bei ausgeheilter Myokarditis ( $\times 200)$. b HE-Färbung bei dilatativer Kardiomyopathie mit ausgedehnten Fibrosen $(\times 200)$. c Immunhistologischer Nachweis CD3-positiver T-Lymphozyten mit fokalem Expressionsmuster bei Autoimmunmyokarditis (rot, × 200). d HE-Färbung bei inflammatorischer Kardiomyopathie mit deutlich vermehrten Entzündungszellen ( $\times 200)$. e In-Situ-Hybridisierung mit Nachweis von Coxsackie-B3-Virus bei Virusmyokarditis. $\mathbf{f ~ H E - F a ̈ r b u n g ~ v i r u s p o s i t i v e r ~ d i l a t a t i v e r ~ i n f l a m m a t o r i s c h e r ~ K a r d i o - ~}$ myopathie $(\mathrm{DCMi})$ mit bereits ausgeprägter Fibrosierung $(\times 200)$. 


\section{Mechanismen}

Eine chronische Myokarditis wird durch verschiedene Mechanismen induziert bzw. aufrechterhalten:

- veränderte virale Replikationsstrategien

- die Unfähigkeit von Effektoren der lokalen Immunität, dauerhaft infizierte myokardiale Zellen zu eliminieren

- die Infektion von Immunzellen selbst

Enteroviren. Die Aufrechterhaltung einer chronischen enteroviralen inflammatorischen Kardiomyopathie ist auf molekularer Ebene am besten untersucht. Das Coxsackie-Virus B3 wird dabei über den Coxsackie-Adenovirus-Rezeptor (CAR) internalisiert, führt zu einem ausgeprägten Remodeling des Myokards und verursacht darüber eine dilatative Kardiomyopathie. Wird der CAR auf kardialen Myozyten nicht exprimiert, bleiben die virale Infektion und die Inflammation des Herzens aus. In explantierten Herzen von Patienten mit dilatativer Kardiomyopathie ist die CAR-Expression deutlich höher als bei anderen Herzerkrankungen oder bei gesunden Herzen. Das angeborene Immunsystem wird durch virale RNA über Toll-like-Rezeptoren aktiviert. Während der MyD88-abhängige Pathway die Aktivierung von „nuclear factor kappa B“ induziert, führt die Stimulation von TRIF primär über eine Aktivierung bestimmter Transkriptionsfaktoren, z. B. „Interferon receptor factors (IRFs)“, zu einer Produktion von Typ-1-Interferonen, die antiviral wirksam sind. Letztlich wird aber eine Kaskade von humoralen und zellulären immunologischen Prozessen initiiert, die vor allem zu einer Viruseliminierung führen soll, sich aber in manchen Fällen im Verlauf auch gegen das myokardiale Gewebe selbst richten kann. Die Aktivierung von Zytokinen (Tumornekrosefaktor $\alpha$ [TNF- $\alpha$ ], Interleukin 1 -6) dient einerseits der Kontrolle der Virusinfektion, kann jedoch andererseits auch die myokardiale Schädigung aggravieren.

\section{Enteroviren: Internalisierung über den} CAR-Rezeptor.

Parvoviren. Im Unterschied zur EV-Infektion befällt die akute parvovirale Infektion des menschlichen Herzens Endothelien kleiner intramyokardialer Arteriolen und postkapillärer Venolen als spezifische Zielzellen. Die endotheliale Infektion induziert die Expression von Adhäsionsmolekülen, gefolgt von einer intravaskulären Akkumulation, Adhäsion und Penetration von immunkompetenten Zellen, die das umgebende Myokard infiltrieren. Die akute B19V-Infektion kann daher mit einer myokardialen Mikrozirkulationsstörung mit Rarifizierung der Kapillardichte [31] einhergehen.
Dementsprechend finden sich bei einem Teil der Patienten initial die klinischen Zeichen eines Myokardinfarkts, aber auch einer nachgewiesenen diastolischen Funktionsstörung [14]. Bei der B19V-Infektion erscheint der Nachweis positiver mRNA als Hinweis auf eine aktive Virusreplikation pathophysiologisch relevant zu sein. Darüber hinaus wurde eine krankheitsspezifische differenzielle Expression von Mikro-RNAs (miRNAs) in der Regulation der Replikation der Viren gefunden. So ergab z.B. die Analyse von Patienten mit latenter und reaktivierter B19V-Infektion 29 differenziell regulierte miRNAs [15]. Dennoch sind die molekularen Mechanismen, die eine isolierte endotheliale und diastolische Dysfunktion erklären könnten, bislang nicht vollständig bekannt. Auch die ätiopathogenetische Bedeutung einer B19-Infektion für die Entwicklung einer möglichen kardialen Dysfunktion ist - insbesondere aufgrund der hohen Durchseuchungsrate mit B19V - bis heute umstritten und Gegenstand der aktuellen Forschung.

Parvovirus B19: endotheliale Infektion kleiner intramyokardialer Arteriolen und postkapillärer Venolen.

Herpesviren. Ähnlich wie beim B19V findet sich in hohem Prozentsatz bei der erwachsenen Bevölkerung nach Primärinfektion in der Kindheit - eine latente HHV-6-Virusinfektion im Myokard. Interessanterweise ist das HHV 6 in der Lage, sein Genom in Telomere von humanen Chromosomen (ciHHV 6) zu integrieren, was eine Übertragung in die Keimlinie bei ca. 0,4-0,8\% der US- und europäischen Bevölkerung bedingt. Ähnlich wie bei anderen Herpesviren werden auch HHV 6 und ciHHV 6 unter (sub-)akuten Bedingungen reaktiviert. Erste Follow-up-Studien weisen auf eine Assoziation von Viruspersistenz und Aggravation des klinischen Verlaufs hin [16].

HHV 6: latente Virusinfektion im Myokard bei vielen Erwachsenen.

\section{Chronische Autoimmunmyokarditis}

\section{Verlauf}

Postinfektiöse chronische Prozesse spielen in der Klinik eine bedeutende Rolle, da sie den Herzmuskel durch persistierende (auto-)zelluläre oder humorale Immunprozesse chronisch progredient schädigen. Der Erreger ist in diesen späteren Krankheitsstadien nicht mehr nachweisbar. Paradoxerweise führt die Aktivierung des angeborenen Immunsystems zu einer verstärkten 
Aktivierung der adaptiven Immunantwort mit Einwanderung von T-Lymphozyten in das zuvor infizierte Myokard. Aktivierte autoreaktive bzw. kreuzreaktive T-Lymphozyten können dann einen chronischen Entzündungsprozess über einen langen Zeitraum aufrechterhalten, auch wenn das Virus bereits eliminiert wurde. Das Gewebe wird dabei mehr und mehr geschädigt und das Matrix-Remodeling breitet sich aus. Dass die kardiale Inflammation den Umbau der extrazellulären Matrix mit einer Kollagenakkumulation fördert und so die linksventrikuläre Funktion beeinflusst, konnte kürzlich in Endomyokardbiopsien von Patienten mit Herzinsuffizienz und dilatativer Kardiomyopathie gezeigt werden [17].

\section{Mechanismen}

Einer der möglichen Mechanismen ist die Transdifferenzierung von Fibroblasten zu Myofibroblasten. Kardiale Fibroblasten sind hiernach proinflammatorische Mediatoren und können durch mechanische Beanspruchung aktiviert werden. Letztlich können somit inflammatorische Zellen und proinflammatorische Zytokine die kardiale Funktion direkt durch eine Veränderung der Myozyten beeinflussen, ferner auch den Umbau der extrazellulären Matrix anregen und auf diesem Weg indirekt die Progression der Erkrankung vorantreiben, was schlussendlich die Entwicklung einer dilatativen Kardiomyopathie induziert.

\section{Klinisches Bild und Diagnostik}

Die klinischen Manifestationen der Myokarditis sind hochvariabel mit einem breiten Spektrum von Symptomen. Die Spanne reicht vom subklinischen Verlauf bis hin zur Präsentation wie bei einem Myokardinfarkt; bedrohliche AV-Blockierungen, tachykarde ventrikuläre Arrhythmien oder eine Herzinsuffizienz sind genauso gut möglich wie ein kardiogener Schock $[2,4]$. Im Gegensatz zum Myokardinfarkt sind EKG-Veränderungen nicht an die Versorgungsgebiete der Koronarien gekoppelt. Erstmals zeigen sich die Symptome oft nach einem viralen Infekt in Form einer mehr oder weniger eingeschränkten Belastbarkeit oder in neu auftretenden Herzrhythmusstörungen.

\section{LV-Funktion}

Die Größe der Ventrikel und die LV-Funktion sind dabei vollständig variabel. Eine noch vollständig erhaltene linksventrikuläre Funktion schließt eine intramyokardiale Entzündungsreaktion nicht aus und ist bei knapp der Hälfte der Patienten mit hochgradigem Verdacht auf eine Myokarditis nachweisbar (unveröffentlichte Daten).

\section{Biomarker}

Biomarker sind trotz fehlender Spezifität für die klinische Diagnose einer Myokarditis hilfreich. Bei einem Teil der Patienten mit akuter Myokarditis besteht ein infarktähnliches klinisches Bild mit akut einsetzenden pektanginösen Beschwerden, ST-Strecken-Hebungen und positivem Troponin. Patienten mit bioptisch gesicherter Myokarditis zeigen signifikant häufiger erhöhte Troponinwerte im Vergleich zur Kreatinkinase. Insbesondere bei Patienten mit akuter Myokarditis lassen sich erhöhte Troponinkonzentrationen im Serum nachweisen.

\section{Empfehlungen für die Praxis [9]}

- Bei allen Patienten mit dem klinischen Verdacht auf eine Myokarditis sollte ein 12-Kanal-Elektrokardiogramm angefertigt werden.

- Troponin, Blutsenkungsgeschwindigkeit und C-reaktives Protein sollten bei allen Patienten zur Beurteilung herangezogen werden.

\section{Echokardiografie}

Unter anderem regionale oder globale Wandbewegungsstörungen, ödembedingte verdickte Ventrikelwände und ein Perikarderguss weisen echokardiografisch auf einen möglichen myokardialen Entzündungsund Schädigungsprozess hin. Die Speckle-TrackingEchokardiografie entwickelt sich hierbei zum neuen diagnostischen Tool - nicht nur bei der koronaren Herzerkrankung, sondern auch bei Patienten mit inflammatorischer Kardiomyopathie. Die globale longitudinale Strain-Analyse kann trotz guter linksventrikulärer Funktion subtile regionale Funktionsstörungen aufzeigen, weist somit auf Pathologien hin und korreliert direkt mit der intramyokardialen Entzündungsreaktion in der Endomyokardbiopsie [18].

Empfehlungen für die Praxis [9]

- Bei jedem Patient mit dem klinischen Verdacht auf eine Myokarditis sollte zu Beginn eine Echokardiografie durchgeführt werden.

- Diese Echokardiografie sollte während des stationären Aufenthaltes wiederholt werden, wenn sich eine hämodynamische Verschlechterung zeigt. 


\section{Kardiale MRT}

Eine Magnetresonanztomografie (MRT) kann bei klinisch stabilen Patienten erwogen werden, ersetzt aber nicht die Endomyokardbiopsie (EMB) und sollte diese nicht verzögern. Zur kardialen MRT der Myokarditis sollten entsprechend der Expertenempfehlung mindestens 2 der 3 Lake-Louise-Kriterien (Ödemdarstellung in der T2-Gewichtung, Early Gadolinium Enhancement in der T1-Gewichtung, Late-GadoliniumEnhancement-Nachweis) herangezogen werden, um die diagnostische Sicherheit und Aussagekraft zu verbessern. Während die Sensitivität der EMB-Ergebnisse bei einer akuten Myokarditis sehr hoch sind (80\%), liegen sie bei der kardialen MRT für eine chronische Myokarditis deutlich niedriger (57\%) [19]. Insbesondere geringe Entzündungsprozesse und vor allem eine Virusinfektion werden durch die bildgebende Diagnostik nicht erfasst. Da diese jedoch entscheidend für einen möglichen Therapiebeginn sind, ist die MRTDiagnostik somit limitiert.

\section{Empfehlungen für die Praxis [9]}

- Ergebnisse aus der MRT sollten sich bei der Diagnose Myokarditis auf die Lake-Louise-Kriterien stützen.

- Eine MRT kann bei klinisch stabilen Patienten neben einer EMB erwogen werden. Sie ersetzt die EMB bei der Diagnose einer Myokarditis aber nicht und sollte die EMB in lebensbedrohlichen Situationen nicht verzögern.

- Eine nuklearmedizinische Untersuchung wird in der Diagnostik einer Myokarditis nicht routinemäßig empfohlen. Ausnahme ist der konkrete Verdachtsfall einer kardialen Sarkoidose.

\section{Endomyokardbiopsie}

Eine ätiologische Diagnostik mit einer möglichen therapeutischen Konsequenz gelingt letztlich nur mit der EMB. Sie ist nach wie vor der Goldstandard für die Diagnose der Myokarditis [20].

Indikation. Eine EMB ist indiziert, wenn andere, sekundäre Ursachen der Herzinsuffizienz wie die koronaren Herzerkrankung oder ein Klappenvitium ausgeschlossen sind. Die Indikation orientiert sich an den Empfehlungen der „American Heart Association“ (AHA), des „American College of Cardiology“ (ACC) und der ESC, die verschiedene Krankheitsszenarien beschreiben [20]. Mit dem aktuellen Konsensuspapier gibt es nun weiterführende eindeutige Empfehlungen der ESC:

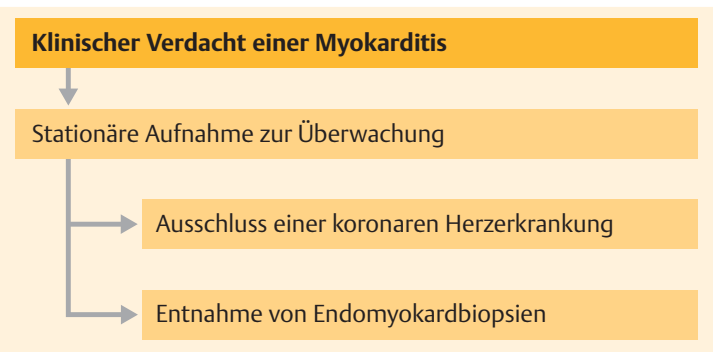

Abb. 2 Flussdiagramm zum diagnostischen Vorgehen bei Patienten mit dem klinischen Verdacht auf eine Myokarditis [9].

Eine selektive Koronarangiografie und eine nachfolgende EMB sind bei allen Patienten mit dem klinischen Verdacht auf eine Myokarditis sinnvoll (Abb.2). Auch hier wurden dazu Krankheitsbilder kategorisiert, um diejenigen Patienten, die eine EMB zur Diagnosesicherung und als Grundlage einer Therapie benötigen, im klinischen Alltag zu identifizieren:

- akute Symptomatik ähnlich dem akuten Koronarsyndrom

- Neuauftreten oder Verschlechterung der Herzinsuffizienz nach Ausschluss einer koronaren Herzerkrankung und bekannter Ursachen für die Herzinsuffizienz

- chronische Herzinsuffizienz nach Ausschluss einer koronaren Herzerkrankung und bekannter Ursachen für die Herzinsuffizienz

- lebensbedrohliche Symptome nach Ausschluss einer koronaren Herzerkrankung und bekannter Ursachen für die Herzinsuffizienz

\section{Empfehlungen fïr die Praxis [9]}

- Alle Patienten mit dem klinischen Verdacht auf eine Myokarditis sollten einer selektiven Koronarangiografie und einer EMB unterzogen werden.

- Die in der EMB gewonnenen Proben sollte histologisch, immunhistologisch und mittels Virus-PCR untersucht werden (dies gilt für Myokardgewebe und Blutproben).

Kontrovers zu diskutieren ist, ob bei Patienten mit der typischen Klinik einer akuten Myokarditis eine Myokardbiopsie erforderlich ist, insbesondere wenn sich die akute Beschwerden rasch zurückgebildet haben und keine LV-Dysfunktion mehr besteht. Wird in diesem Fall auf eine Biopsie verzichtet, sollte die Myokardfunktion in jedem Fall langfristig echokardiografisch kontrolliert werden, um eine spätere hämodynamische Verschlechterung, die dann eine EMB nach sich ziehen sollte, zu erfassen. 
Sicherheit. Eine große, teils retrospektiv $(\mathrm{n}=2415)$, teils prospektiv ( $n=496)$ angelegte Studie mit knapp 30000 Gewebeentnahmen zeigte praktisch keine schwerwiegende Komplikation bei der Biopsie [21]. Um den „sampling error“ so niedrig wie möglich zu halten, sollte man so viele Proben wie möglich und diese früh im Verlauf der Erkrankung entnehmen. Dies ist insbesondere für die akute Myokarditis relevant, da obwohl bei fulminanten Entzündungsprozessen schon in diesem frühen Stadium großflächige konfluierende Entzündungszellinfiltrate vorliegen können - in den ersten Erkrankungswochen häufiger auch eine fokale Zellinfiltration beobachtet wird.

EMB im linken oder rechten Ventrikel. Simultanstudien belegen, dass die Diagnose durch eine links- und eine rechtsventrikuläre EMB gleich gut möglich ist, weil sich intramyokardiale Inflammation und Virusnachweis nicht signifikant in beiden Ventrikeln unterscheiden. Einzig morphologische Veränderungen lassen sich durch eine LV-Endomyokardbiopsie signifikant häufiger abbilden. Hinsichtlich der Reduktion des „sampling errors“ sind (mindestens) 3 Endomyokardbiopsien notwendig, um die „wahre“ Diagnose stellen, also das Virus bei akuter und chronischer Myokarditis nachweisen zu können [22].

\section{Empfehlungen fïr die Praxis [9]}

- Mindestens 3 Myokardproben, jede von 1-2 mm Größe, sollten (vom rechten oder linken Ventrikel) entnommen und sofort in $10 \%$ igem Formalin bei Raumtemperatur für die Lichtmikroskopie fixiert werden; zusätzlich sollten Proben schockgefroren in Flüssigstickstoff bei $-80^{\circ} \mathrm{C}$ oder in RNA-haltigen Röhrchen bei Raumtemperatur für die Virus-PCR gelagert werden.

- Eine EMB kann, wenn nötig, wiederholt werden, um eine ätiologiegerichtete Therapieantwort zu steuern oder wenn ein „sampling error“ bei Patienten mit unklarem Progress der Herzinsuffizienz angenommen wird.

Molekularpathologie und Virologie. Virusvermehrung und immunpathogenetische Mechanismen spielen ätiologisch eine wesentliche Rolle. Die molekulare Virusdiagnostik ist aber nicht nur auf den reinen Nachweis erregerspezifischer Nukleinsäuren beschränkt, sondern beinhaltet auch die Quantifizierung der Viruslast mittels Realtime-PCR, die Sequenzierung der erhaltenen Genprodukte zur Bestimmung des Virussubtyps und die Replikation. Ob Viruslast, die im Myokard nachweisbaren Virussubtypen und der Nachweis von
Replikation prognostisch bedeutsam sind, ist bislang nicht vollständig geklärt. Während bei der CoxsackieAdenovirus -Infektion des Myokards der Virusnachweis per se pathologisch bedeutsam ist, ist dies beim B19V bislang ungeklärt. Oft verursachen Viren jedoch eine stärker symptomatische Erkrankung mit deutlicherer Gewebeverletzung, wenn sie reaktiviert werden.

Antivirale Antikörper. Ein serologischer Nachweis von antiviralen Antikörpern (Ausnahme Hepatitis C und HIV) wird nicht empfohlen, weil die Untersuchung hinsichtlich Sensitivität und Spezifität in der Diagnosestellung nur eine begrenzte Aussagekraft hat.

\section{Empfehlungen für die Praxis [9]}

- Eine routinemäßige Virus-Serumdiagnostik wird nicht empfohlen.

- Serumproben sollten, wenn möglich, auf kardiale Autoantikörper untersucht werden, wenn ein (oder mehrere) veröffentlichte Tests zur Verfügung stehen, gerichtet nach der spezifischen Zentrumsexpertise. Krankheitsspezifische Autoantikörper sollten dabei vorzugsweise getestet werden.

Histologie und Immunhistologie. Nach den Dallas-Kriterien ist die akuteMyokarditis gesichert, wenn Myozytennekrosen,umgeben von mononukleären Zellinfiltraten, nachgewiesenwerden. Die Diagnose einer Borderline-Myokarditiswird gestellt, wenn Zellnekrosen fehlen, aberinflammatorische Infiltrate vorhanden sind. Die Beurteilungder EMB nach den Dallas-Kriterien wird durchdie Tatsache eingeschränkt, dass nichtzelluläre inflammatorischeProzesse nicht nachgewiesen werden können. Der Nachweis von CD3- und LFA-1positiven Lymphozyten, CD45R0-positiven T-Memoryzellen, Mac-1-positiven Makrophagen, Perforin-positiven zytotoxischen Zellen sowie zusätzlich eine verstärkte endotheliale Expression von Zelladhäsionsmolekülen (z. B. HLA-1 = „human leukocyte antigen 1“, ICAM-1 = ,intercellular adhesion molecule 1“, VCAM-1 = „Vascular cell adhesion protein 1“) erlauben eine sichere Diagnose auch einer chronischen myokardialen Entzündungsreaktion mit diffusen Zellinfiltrationen. Durch diese zusätzliche immunhistologische Untersuchung steigt die Anzahl der positiven pathologischen Endomyokardbefunde deutlich an. 
Abb. 3 Perforin in der EMB ist stärkster Prediktor für eine LV-Funktionsverschlechterung bei Patienten mit inflammatorischer Kardiomyopathie im Langzeitverlauf [26]. Univariable Analyse zwischen $\triangle$-LVEF (Differenz zwischen Baseline LVEF und Follow-up LVEF in \%) und der Perforinexpression (Zellen $/ \mathrm{mm}^{2}$ ).

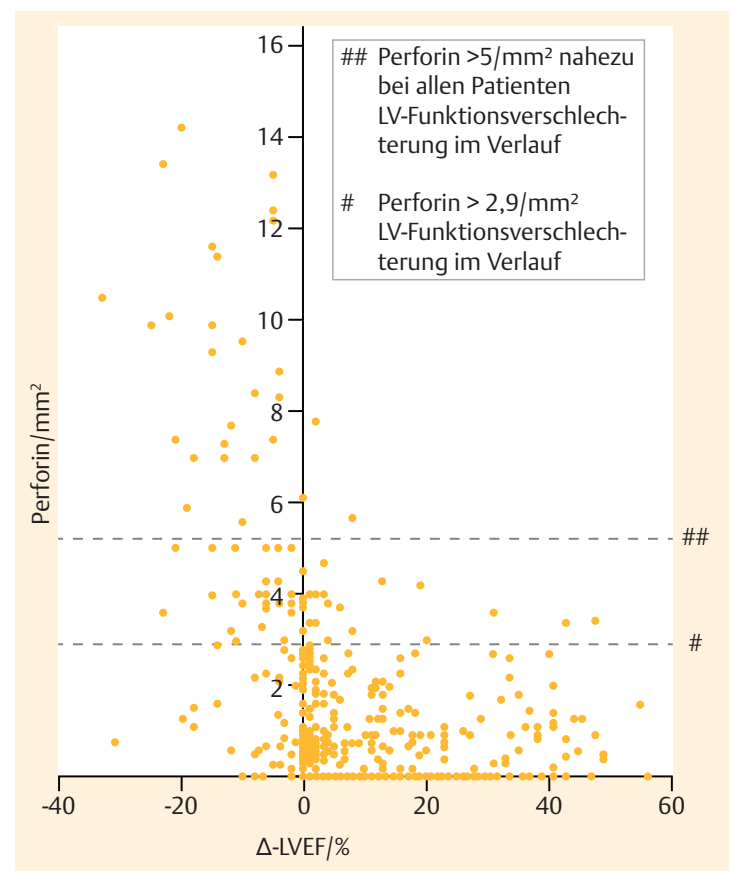

\section{Epidemiologie und Prognose}

In über 70\% der Fälle kann eine akute Myokarditis in den ersten 4 Wochen folgenlos abheilen. Bei ungefähr $25 \%$ der Patienten, die zum Zeitpunkt der Erstdiagnose bisher nicht abgrenzbar sind, kommt es zu einer klinischen Verschlechterung, z.T. bis zur akuten Dekompensation oder der Progredienz der Herzinsuffizienz in Form einer dilatativen Kardiomyopathie. Es konnte zudem gezeigt werden, dass im Langzeitverlauf 3 Jahre nach akuter Myokarditis - 49\% der Patienten trotz normalisierter LV-Funktion (HFNEF $=$, heart failure with normal ejection fraction“) eine Herzinsuffizienzsymptomatik beklagten - assoziiert mit der Entwicklung einer diastolischen Funktionsstörung [23].

Initiale Herzinsuffizienz. Besteht allerdings bereits initial eine deutliche, im Verlauf progrediente Herzinsuffizienz, ist die langfristige Prognose erheblich eingeschränkt [24]. Die 5-Jahres-Mortalität wird für diese Patientengruppe mit 25-46\% angegeben.

Entzündungsnachweis. Dass der immunhistochemische Nachweis einer intramyokardialen Entzündungsreaktion per se-und nicht der alleinige PCR-Nachweis viraler Genome - mit einer schlechten Prognose einhergeht, wurde in einer Langzeituntersuchung bei 181 Patienten mit Verdacht auf eine entzündliche Herzmuskelerkrankung gezeigt [25]. Die intramyokardiale Entzündung stellte hier einen unabhängigen Prädiktor hinsichtlich Morbidität und Mortalität dar.
In Untersuchungen konnte belegt werden, dass bei bis zu $60 \%$ der Patienten mit dem klinischen Bild einer dilatativen Kardiomyopathie eine (virusassoziierte) chronische Entzündung nachgewiesen werden konnte.

Perforin als prognostischer Marker. Die vermehrte Expression des porenformenden Proteins Perforin, das von Natürliche-Killer-Zellen, $\gamma \delta$-Zellen und zytotoxischen T-Lymphozyten gebildet und intrazellulär gespeichert wird, scheint prognostisch eine wesentliche Rolle zu spielen [26]. In einer Studie an 495 virusnegativen Patienten konnte Perforin in der Endomyokardbiopsie multivariat als stärkster Prädiktor für die hämodynamische Verschlechterung im Langzeitverlauf determiniert werden. Der berechnete optimale Cut-offWert für Perforin-positive Zellen in der $\mathrm{EMB} / \mathrm{mm}^{2}$ betrug dabei 2,95 (mit einer 94,2\%igen Sensitivität und einer 80,4\%igen Spezifität) für ein hohes Risiko einer LV-Funktionsverschlechterung (Abb.3).

\section{Empfehlungen für die Praxis [9]}

- Alle Patienten mit einer Myokarditis sollten sowohl klinisch, mit EKG und mit Echokardiografie nachverfolgt werden.

- Eine Langzeitverlaufsuntersuchung wird für Patienten nach stattgehabter Myokarditis empfohlen.

Riesenzellmyokarditis. Für die Riesenzellmyokarditis ist die Prognose noch wesentlich schlechter. Unbehandelt verläuft sie nahezu immer infaust und ist - neben einer nekrotisierenden eosinophilen Myokarditis eine Notfallindikation zur EMB.

- Die Riesenzellen sind zwar charakteristisch, können aber aufgrund des hohen „sampling errors“ übersehen werden, auch wenn zahlreiche Schnittebenen untersucht werden.

- Der histologische Befund einer kardialen Sarkoidose als häufigster Form einer granulomatösen Myokarditis gleicht dem der Riesenzellmyokarditis: Wie bei einer extrakardialen Sarkoidose kommen dabei nicht nekrotisierende Granulome vor, die das Myokard ersetzen. Eine neue Möglichkeit der Differenzierung bietet hier die Genexpressionsanalyse, da bei beiden Erkrankungen unterschiedliche Gene inflammatorischer Prozesse als differenziell reguliert nachgewiesen werden konnten (Abb.4) [27]. 
Viruspersistenz. Während eine Persistenz von Coxsackie-/Adenovirus bekanntermaßen mit einer LV-Funktionsverschlechterung einhergeht, gibt es Hinweise darauf, dass auch der Nachweis von Entzündung und B19V-Replikation mit einer schlechteren Prognose einhergeht, wobei die Viruslast selbst keine Bedeutung zu spielen scheint (data submitted).

\section{Empfehlungen fïr die Praxis [9]}

Patienten mit lebensbedrohlichem Erscheinungsbild sollten in spezialisierte Zentren mit verfügbarem hämodynamischem Monitoring, Herzkatheter und Expertise für eine EMB gebracht werden.

\section{Therapeutische Implikationen}

Die Behandlung einer Myokarditis hängt von der klinischen Symptomatik, dem Erkrankungsverlauf und vor allem von den Ergebnissen der EMB ab.

Eingeschränkte LV-Funktion. Bei eingeschränkter linksventrikulärer Pumpfunktion sollte gemäß den aktuellen Leitlinien eine evidenzbasierte medikamentöse Herzinsuffizienztherapie mit ACE-Hemmer, Betablocker, Aldosteronrezeptorantagonist und Schleifendiuretikum eingeleitet werden. Die Betablockergabe zeigte im murinen Modell eine Suppression inflammatorischer Zytokine durch Carvedilol. Bei Patienten mit fulminanter Myokarditis und akuter dekompensierter Herzinsuffizienz liegen keine Daten vor, die den Nutzen einer Betablockertherapie belegen. Allerdings haben Patienten mit schwerer Herzinsuffizienz (NYHA-Klasse III - IV) und positiver Immunhistologie ohne Betablockertherapie eine schlechte Prognose mit einer 5-Jahre-Überlebensrate von nur 39\%.

Herzrhythmusstörungen. In der akuten Phase der Myokarditis sind - gerade bei hochgradig eingeschränkter LV-Funktion - maligne Herzrhythmusstörungen nicht selten. Eine ICD-Implantation sollte in der akuten Episode zurückgestellt werden, da von einer Besserung auszugehen ist. Alternativ kann das Anlegen einer LiveVest zur Überbrückung des akuten Stadiums erwogen werden.

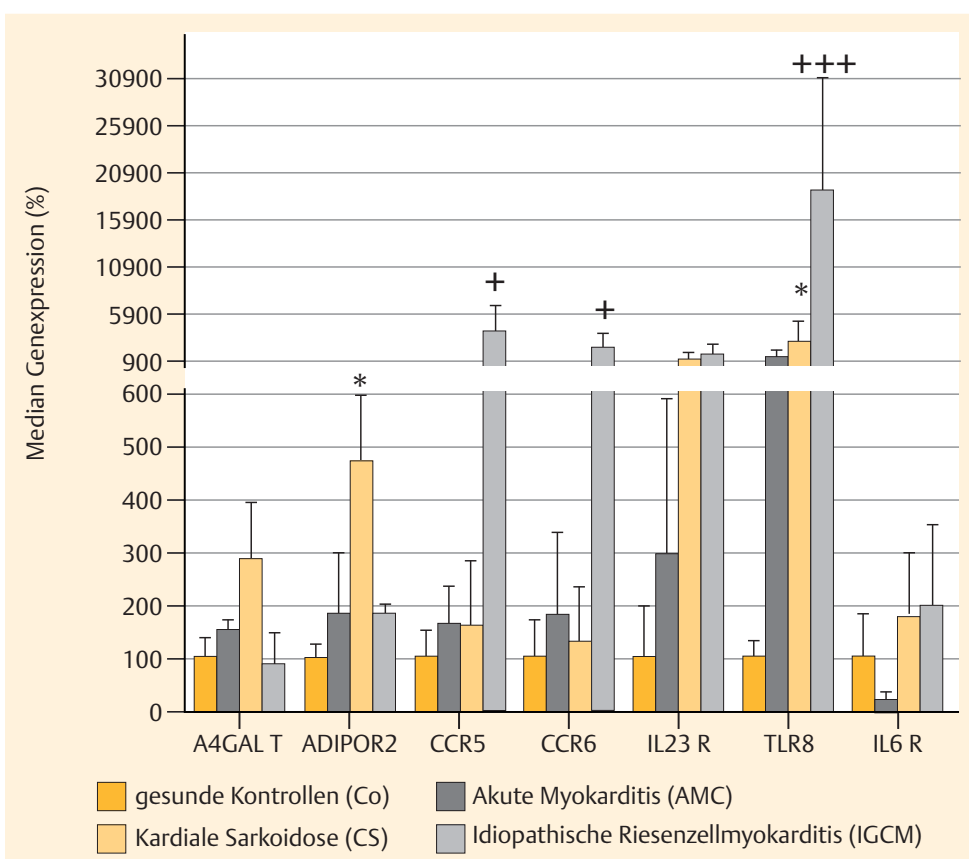

Abb.4 Beispiel einer differenziellen Genregulation von zellulären und Toll-Like Rezeptoren bei akuter Myokarditis, kardialer Sarkoidose und Riesenzellmyokarditis im Vergleich zu gesunden Kontrollen. CS vs. Co: ${ }^{*} \mathrm{P}<0,05$; IGCM vs. Co: $+\mathrm{P}<0,05 ;+++\mathrm{P}<0,001$ (modifiziert nach [27]).

\section{Empfehlungen für die Praxis [9]}

- Das Management der ventrikulären Dysfunktion sollte den ESCGuidelines für die Herzinsuffizienz entsprechen.

- Das Management für Arrhythmien außerhalb einer akuten Phase sollte den ESC-Guidelines für Arrhythmien entsprechen.

- Eine ICD-Implantation sollte zurückgestellt werden, bis die akute Episode beendet ist.

Sportkarenz. Bei Patienten mit akuter Myokarditis ist Sport kontraindiziert. Sportler mit vermuteter bzw. gesicherter Herzmuskelentzündung sollten über einen Zeitraum von mindestens 6 Monaten nicht am Wettkampfsport teilnehmen. Das Training sollte erst wieder aufgenommen werden, wenn sich die linksventrikuläre Pumpfunktion normalisiert hat und kein Anhalt für klinisch relevante Arrhythmien besteht. 


\section{Empiehlungen fitr die Praxis [9]}

Körperliche Aktivität sollte während der akuten Phase und für mindestens 6 weitere Monate bei Sportlern und Nicht-Sportlern eingeschränkt werden. Diese Empfehlungen basieren auf einer Expertenmeinung der Arbeitsgruppe.

Hämodynamisch instabile Patienten. Bei trotz optimaler medikamentöser Therapie hämodynamisch instabilen Patienten mit fulminanter Myokarditis muss die Implantation eines mechanischen Kreislaufunterstützungssystems als „Bridging“ bis zur Erholung der linksventrikulären Pumpfunktion oder zur Herztransplantation diskutiert werden. Trotz der initial dramatisch erscheinenden klinischen Präsentation haben diese Patienten eine Überlebenswahrscheinlichkeit von $60-80 \%$.

\section{Empfehlungen für die Praxis [9]}

- Bei hämodynamisch instabilen Patienten kann ein kardiopulmonales Assist-Device als „Bridging“ zur hämodynamischen Verbesserung oder vor einer Herztransplantation dienen.

- In der akuten Phase sollte eine Herztransplantation zurückgestellt werden, da noch eine Verbesserung eintreten kann. Sie kann aber für den hämodynamisch instabilen Myokarditis-Patienten, auch mit einer Riesenzellmyokarditis, in Betracht gezogen werden, wenn eine optimale pharmakologische Therapie und mechanische Unterstützung den Patienten nicht stabilisieren kann.
Immunsuppressive Therapie. Eine immunsuppressive Therapie ist bei Patienten mit Riesenzellmyokarditis, eosinophiler Myokarditis und Sarkoidose klar indiziert. Dabei hängt das Überleben bei einer Riesenzellmyokarditis davon ab, dass diese Therapie frühzeitig eingeleitet wird. Basierend auf der Diagnostik der EMB zeigen mehrere Studien bei der chronischen, virusnegativen Myokarditis und den Autoimmunmyokarditiden klar positive Ergebnisse (signifikante Verbesserung der Herzinsuffizienzsymptomatik und der LVEF) einer immunsuppressiven Therapie über bis zu 6 Monate mit Steroiden, Azathioprin und Ciclosporin allein oder jeweils in Kombination.

In der TIMIC-Studie an 85 randomisierten Patienten mit einer Herzinsuffizienz $>6$ Monate, $\mathrm{LVEF} \leq 45 \%$ und bioptisch nachgewiesener Myokarditis betrug die Nonresponderrate nach 6-monatiger Therapie mit Prednisolon und Azathioprin nur 12\%, während in der Kontrollgruppe bei keinem Patienten eine Besserung erzielt wurde [28]. Allerdings zeigte eine retrospektive Analyse klar, dass Patienten mit inflammatorischer Kardiomyopathie und Viruspersistenz Therapieversager waren oder sich sogar im klinischen Befund eher verschlechterten [29]. Diese Daten bestätigen die Hypothese, dass bei Patienten mit Viruspersistenz eine Immunsuppressionstherapie die körpereigene antivirale Immunreaktion inhibiert und somit die Viruselimination verhindert. Die positiven Effekte bestätigen sich auch in Langzeituntersuchungen, wonach die hämodynamische Verbesserung der LV-Funktion nach stattgehabter 6-monatiger Immunsuppressionstherapie mit Methylprednisolon und Azathioprin auch im Langzeit-Follow-up-Untersuchungszeitraum von 35,45 $\pm 127,9$ Monaten anhielt, bzw. eine sich entwickelnde kardiale Dysfunktion nicht weiter fortgeschritten ist (Escher et al. unveröffentlichte Daten).

\section{Empfehlungen fitr die Praxis [9]}

- Mit einer Immunsuppression sollte erst begonnen werden, wenn eine aktive Virusinfektion in der EMB mittels PCR ausgeschlossen wurde.

- Basierend auf der Erfahrung bei nicht kardialen Autoimmunerkrankungen empfiehlt die Arbeitsgruppe eine eventuelle Immunsuppression, wenn eine Autoimmunmyokarditis nachgewiesen wurde und eine Immunsuppression nicht kontraindiziert ist - inkl. einer Riesenzellmyokarditis, einer kardialen
Sarkoidose und einer Myokarditis, die mit einer nicht kardialen Autoimmunerkrankung assoziiert ist.

- Eine Steroidtherapie ist bei kardialer Sarkoidose indiziert, wenn eine ventrikuläre Dysfunktion und/oder Arrhythmien bestehen und bei einigen Formen nicht infektiöser eosinophiler oder toxischer Myokarditis mit Herzinsuffizienz und/oder Arrhythmien.
- Eine Immunsuppression kann individuell in Betracht gezogen werden bei Patienten mit nicht infektiöser lymphozytärer Myokarditis, die therapierefraktär zur Standardmedikation ist, wenn die Immunsuppression nicht kontraindiziert ist.

- Eine Verlaufs-EMB kann erforderlich sein, um die Dosierung und die Dauer einer Immunsuppression zu steuern. 
Die Gabe von Immunglobulinen verbesserte bislang nur bei Kindern mit akuter Myokarditis die kardiale Funktion und die 1-Jahr-Überlebensrate.

Antivirale Therapie. Bei chronischen EV- und ADVInfektionen führt die Behandlung mit $8 \times 10^{6} \mathrm{U}$ Interferon- $\beta$ über 6 Monate zu einer effektiven Viruselimination (Abb.5) [30]. Die therapeutischen Effekte von Interferon- $\beta$ werden auf eine Verstärkung der antiviralen Immunreaktion zurückgeführt. Die Behandlung wird als nebenwirkungsarm toleriert. Bei anderen viralen Infektionen, z. B. B19V, werden verschiedene antivirale Substanzen derzeit hinsichtlich ihrer Wirkdosis und Therapiedauer untersucht. Aufgrund fehlender größerer und kontrollierter Studien können zum gegenwärtigen Zeitpunkt noch keine allgemeingültigen Empfehlungen gegeben werden, für welche Patientengruppen sich ein Behandlungsvorteil ergibt.

Interessenkonflikt: Der korrespondierende Autor gibt für sich und seine Koautoren an, dass kein Interessenkonflikt besteht.

Abstract
Myocarditis is an inflammatory disease of the cardiac
muscle caused by myocardial infiltration of immuno-
competent cells following any kind of cardiac injury.
Myocarditis is a challenging diagnosis due to the hete-
rogeneity of clinical presentations. It can be caused by
infections, drugs, toxic substances, and autoimmune
diseases. Chronic inflammatory events may survive
successful clearance of initial cardiotoxic agents, be
triggered or amplified by autoimmunological proces-
ses, or develop in the context of systemic diseases.
Progression of myocarditis to its sequela, dilated car-
diomyopathy, has been documented in $25 \%$ of cases
and is pathogenically linked to chronic inflammation
and viral persistence. Therapeutic decisions must be
based on the results of endomyocardial biopsy studies
while taking account of the individual patient's clinical
course. Moreover, specific immunosuppressive and
antiviral treatment can help only if a treatable cause is
present (e. g., a viral infection, or inflammatory pro-
cess), and only if the myocardium still has regenerative
potential. Once irreversible myocardial injury has oc-
curred then the development or progression of heart
failure in the long term can no longer be prevented. The
aims are to bridge the gap between clinical and tissue-
based diagnosis, to improve management of aetiology-
driven treatment in inflammatory heart muscle disease.

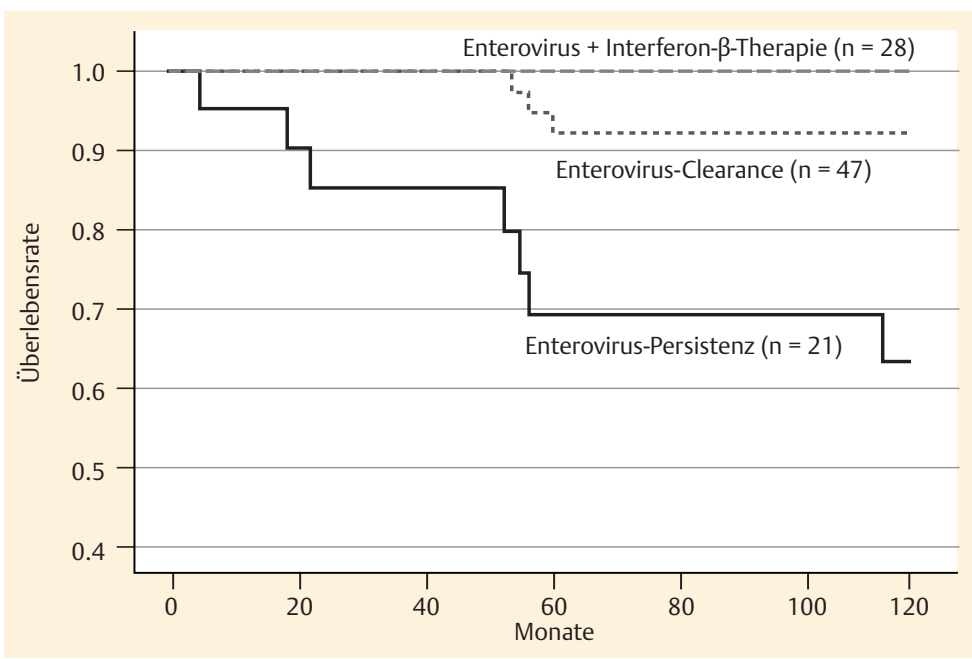

Abb.5 Mortalitätsrate bei Patienten mit Enterovirusinfektion. Dargestellt ist die Überlebensrate von unbehandelten Patienten mit Viruspersistenz in der Follow-up EMB, unbehandelten Patienten mit Virus-Clearance in der Follow-up-EMB und Patienten, die mit Interferon- $\beta$ behandelt wurden (modifiziert nach [30]).

$\begin{array}{ll}\text { Kernaussagen } & \\ \text { - Bei V.a. Myokarditis/inflamma- } & \text { - Eine spezifische Therapie stellt } \\ \text { torische Kardiomyopathie ist } & \text { bei virusnegativen Patienten die } \\ \text { eine EMB klar indiziert. } & \text { Immunsuppression, bei Enterovi- } \\ \text { - Eine exakte Analyse der EMB } & \text { rus-positiven Patienten die Inter- } \\ \text { mittels Molekularbiologie, Histo- } & \text { feron-b-Therapie dar. } \\ \text { logie und Immunhistologie mit } & \text { - Bei fulminantem Verlauf mit V.a. } \\ \text { Charakterisierung und Quantifi- } & \text { Riesenzellmyokarditis ist die EMB } \\ \text { zierung von entzündlichen Infil- } & \text { eine Notfallindikation, eine Gen- } \\ \text { traten ist entscheidend. } & \text { expressionanalyse bietet in die- } \\ & \text { sem Fall ein neues diagnostisches } \\ & \text { Tool. }\end{array}$

\section{Über die Autoren}

\section{Felicitas Escher}

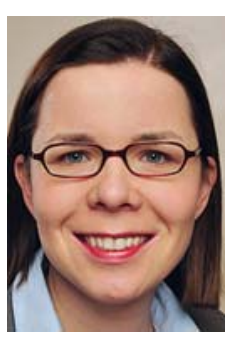

Dr. med. 1996 - 2002 Studium der Humanmedizin in Berlin und Zürich. 2004- 2014 wissenschaftliche Mitarbeiterin in der Klinik für Kardiologie und Pulmologie am Campus Benjamin Franklin der Charité - Universitätsmedizin Berlin. Seit 2003 Beraterin im Institut für Kardiale Diagnostik und Therapie (IKDT) Berlin. 2012/2014 Fachärztin für Innere Medizin und Kardiologie. Seit 2014 Funktionsoberärztin der Klinik für Kardiologie Campus Virchow Klinikum der Charité - Universitätsmedizin Berlin. 


\section{Carsten Tschöpe}

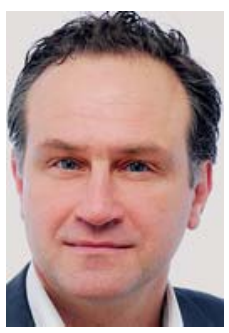

Prof. Dr. med. 1986 - 1993 Studium der Humanmedizin in Heidelberg. 2003 Habilitation für das Fach Innere Medizin. 2007-2014 Stellvertretender Klinikdirektor und leitender Oberarzt der Klinik für Kardiologie und Pulmologie am Campus Benjamin Franklin der Charité - Universitätsmedizin Berlin. Seit 2009 W2-Univ.-Professur am Translationszentrum „Berlin-Brandenburger Zentrum für Regenerative Therapien (BCRT)“. Seit 2014 Stellvertretender Klinikdirektor der Klinik für Kardiologie Campus Virchow Klinikum der Charité - Universitätsmedizin Berlin.

\section{Heinz-Peter Schultheiss}

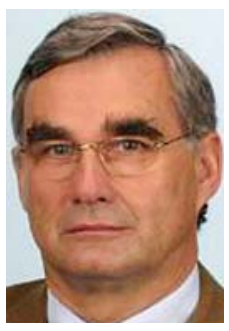

Prof. Dr. med. 1969 - 1974 Studium der Humanmedizin in Freiburg und München; 1986 Habilitation für das Fach Innere Medizin. 1987 - 1989 Heisenberg-Stipendiat der Deutschen Forschungsgemeinschaft; 1989 Berufung als C3-Professor an die HeinrichHeine-Universität Düsseldorf. 1994 2014 Direktor der Klinik für Kardiologie und Pulmologie am Campus Benjamin Franklin der Charité - Universitätsmedizin Berlin. Seit 2014 Emeritus; Leiter des Instituts für Kardiale Diagnostik und Therapie (IKDT) Berlin.

\section{Korrespondenzadresse}

Dr. Felicitas Escher

Charité-Universitätsmedizin Berlin

Klinik für Kardiologie

Campus Virchow Klinikum

Augustenburger Platz 1

13353 Berlin

E-Mail: felicitas.escher@charite.de

\section{Literatur}

1 Caforio AL, Calabrese F, Angeline A et al. A prospective study of biopsy-proven myocarditis: prognostic relevance of clinical and aetiopathogenetic features at diagnosis. Eur Heart J 2007; 28: $1326-1333$

2 Sagar S, Liu PP, Cooper LT. Myocarditis. Lancet 2013; 379: $738-747$

3 Cooper LT, Baughman KL, Feldman AM et al. The role of endomyocardial biopsy in the management of cardiovascular disease. Eur Heart J 2007; 28: 3076 - 3093
4 Schultheiss HP, Kuehl U, Cooper LT. The Management of Myocarditis. Eur Heart J 2011; 32: 2616-2665

5 Aretz HT. Myocarditis: the Dallas criteria. Hum Pathol 1987; 18: $619-624$

6 Thiene G, Bruneval P, Veinot J et al. Diagnostic use of the endomyocardial biopsy: a consensus statement. Virchows Arch 2013; 463: 1 - 5

7 Felker GM, Thompson RE, Hare JM et al. Underlying causes and longterm survival in patients with initially unexplained cardiomyopathy. N Engl J Med 2000; 342: 1077 - 1084

8 Richardson P, McKenna W, Bristow M et al. Report of the 1995 World Health Organization/International Society and Federation of Cardiology Task Force on the Definition and Classification of cardiomyopathies. Circulation 1996; 93: 841-842

9 Caforio AL, Pankuweit S, Arbustini E et al. Current state of knowledge on aetiology, diagnosis, management, and therapy of myocarditis: a position statement of the European Society of Cardiology Working Group on Myocardial and Pericardial Diseases. Eur Heart J 2013; 34: 2636-2648

10 Kühl U, Pauschinger M, Noutsias M et al. High prevalence of viral genomes and multiple viral infections in the myocardium of adults with "idiopathic" left ventricular dysfunction. Circulation 2005; 111: 887-893

11 Pauschinger M, Doerner A, Kuehl U et al. Enteroviral RNA replication in the myocardium of patients with left ventricular dysfunction and clinically suspected myocarditis. Circulation 1999; 99: 889-895

12 Bowles NE, Ni J, Kearney DL et al. Detection of viruses in myocardial tissues by polymerase chain reaction. evidence of adenovirus as a common cause of myocarditis in children and adults. J Am Coll Cardiol 2003; 42: 466-472

13 Kühl U, Lassner D, Pauschinger M et al. Prevalence of erythrovirus genotypes in the myocardium of patients with dilated cardiomyopathy. J Med Virol 2008; 80: 1243-1251

14 Tschöpe C, Bock CT, Kasner M et al. High prevalence of cardiac parvovirus B19 infection in patients with isolated left ventricular diastolic dysfunction. Circulation 2005; 111: 879-886 Epub 2005 Feb 14

15 Kühl U, Rohde M, Lassner D et al. miRNA as activity markers in Parvo B19 associated heart disease. Herz 2012; 37: 637 - 643

16 Kühl U, Lassner D, Wallaschek $\mathrm{N}$ et al. Chromosomally integrated human herpesvirus 6 in heart failure: prevalence and treatment. Eur J Heart Fail 2015; 17: 9-19

17 Lindner D, Zietsch C, Tank J et al. Cardiac fibroblasts support cardiac inflammation in heart failure. Basic Res Cardiol 2014; 109: 428

18 Escher F, Kasner M, Kühl U et al. New echocardiographic findings correlate with intramyocardial inflammation in endomyocardial biopsies of patients with acute myocarditis and inflammatory cardiomyopathy. Mediators Inflamm 2013: 875420

19 Francone $\mathrm{M}$, Chimenti $\mathrm{C}$, Galea $\mathrm{N}$ et al. CMR sensitivity varies with clinical presentation and extent of cell necrosis in biopsyproven acute myocarditis. J Am Coll Cardiol 2014; 7: 254-263

20 Cooper LT, Baughman KL, Feldman AM et al. The role of endomyocardial biopsy in the management of cardiovascular disease: a scientific statement from the American Heart Association, the American College of Cardiology, and the European Society of Cardiology. Endorsed by the Heart Failure Society of America and the Heart Failure Association of the 
European Society of Cardiology. J Am Coll Cardiol 2007; 50: $1914-1931$

21 Holzmann M, Nicko A, Kühl U et al. Complication rate of right ventricular endomyocardial biopsy via the femoral approach. Circulation 2008; 118: 1722 - 1728

22 Escher F, Lassner D, Kühl U et al. Analysis of Endomyocardial Biopsies in suspected Myocarditis - Diagnostic Value on Left versus Right Ventricular Biopsy. Int J Cardiol 2014; 177: 76 78

23 Escher F, Westermann D, Gaub R et al. Development of diastolic heart failure in a 6-year follow-up study in patients after acute myocarditis. Heart 2011; 97: 709-714

24 Magnani JW, Danik HJ, Dec GW Jr et al. Survival in biopsyproven myocarditis: a long-term retrospective analysis of the histopathologic, clinical, and hemodynamic predictors. Am Heart J 2006; 151: $463-470$

25 Kindermann I, Kindermann M, Kandolf R et al. Predictors of outcome in patients with suspected myocarditis. Circulation 2008; 118: 639-648

26 Escher F, Kühl U, Lassner D et al. Presence of Perforin in Endomyocardial Biopsies of Patients with Inflammatory Cardiomyopathy Predicts Poor Outcome. Eur J Heart Fail 2014; 16: $1066-1072$
27 Lassner D, Kühl U, Siegismund CD et al. Improved diagnosis of idiopathic giant cell myocarditis and cardiac sarcoidosis by myocardial gene expressing profil. Eur Heart J 2014: DOI: 10.1093/eurheartj/ehu101

28 Frustaci A, Russo MA, Chimenti C. Randomized study on the efficacy of immunosuppressive therapy in patients with virusnegative inflammatory cardiomyopathy: the TIMIC study. Eur Heart J 2009; 30: 1995-2002

29 Frustaci A, Chimenti C, Calabrese F et al. Immunosuppressive therapy for active lymphocytic myocarditis: virological and immunologic profile of responders versus nonresponders. Circulation 2003; 107: 857-863

30 Kuehl U, Lassner D, von Schlippenbach J et al. Interferon-betaImproves Survival in Enterovirus-Associated Cardiomyopathy. J Am Coll Cardiol 2012; 60: 1295-1296

31 Schmidt-Lucke C, Zobel S, Schrepfer S et al. Impaired endothelial regeneration through human parvovirus B19-infected circulating angiogenic cells in cardiomyopathy. JID 2015 (in press) 


\section{CME。thieme.de}

\section{CME-Fragen}

\section{CME-Teilnahme}

- Viel Erfolg bei lhrer CME-Teilnahme unter http://cme.thieme.de

- Diese Fortbildungseinheit ist 12 Monate online für eine CME-Teilnahme verfügbar.

- Sollten Sie Fragen zur Online-Teilnahme haben, unter http://cme.thieme.de/hilfe finden Sie eine ausführliche Anleitung.

1 Welches der folgenden Symptome kann zum klinischen Bild einer Myokarditis gehören?

A ventrikuläre Tachykardien

B Herzinsuffizienz

C kardiogener Schock

Deichen eines Myokardinfarkts

E alle Symptome

2 Was trifft auf die kardiale Bildgebung bei der Myokarditis nicht zu?

A Die MRT-Diagnostik sollte sich auf die Lake-Louise-Kriterien stützen.

B Das MRT ersetzt nicht die EMB-Diagnostik.

C Bei der chronischen Myokarditis hat die MRT eine hohe Sensitivität und Spezifität.

Eine auffällige transthorakale Echokardiografie schließt eine intramyokardiale Entzündungsreaktion nicht aus.

In der Speckle-Tracking-Analyse korreliert der globale longitudinale Strain mit der intramyokardialen Entzündung.

3 Welche der folgenden Aussagen zur Myokarditis ist nicht richtig?

A Eine Virusdiagnostik gelingt mittels PCR-Analyse.

B Eine Myokarditis ist histologisch durch den Nachweis von entzündlichen Infiltraten, assoziiert mit Myozytendegeneration und Nekrosen definiert.

C Der virusserologische Befund korreliert häufig mit dem molekularbiologischen Virusgenomnachweis im Myokard.

D Erythroviren sind die heute am häufigsten nachgewiesenen Viren.

E Eine Virusmyokarditis ist von einer Autoimmunmyokarditis abzugrenzen.

$4 \quad$ Welcher der folgenden Parameter bei der Myokarditis-Therapie hat keine prognostische Aussagekraft?

A Nachweis intramyokardialer Inflammation in der EMB

B Betablocker

C Nachweis von Enterovirusgenom im Myokard

D Parvovirus- $\mathrm{B}_{19}$-Serologie

E erhöhte intramyokardiale Perforinexpression

5 Welche der folgenden Aussagen zur Riesenzellmyokarditis ist nicht richtig?

A Die Riesenzellmyokarditis ist unbehandelt mit einer hohen Mortalität behaftet.

B Histologisch zeigt sich das Bild mehrkerniger Riesenzellen mit einer massiven Destruktion von Myokardzellen.

C Eine Differenzierung zur Sarkoidose ist histologisch einfach.

D Die EMB stellt bei der Riesenzellmyokarditis eine Notfallindikation dar.

E Eine Differenzierung zur Sarkoidose ist mittels Genprofilanalyse möglich. 


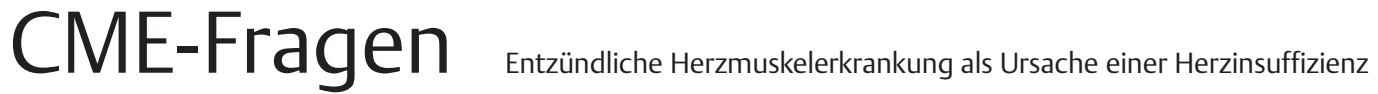

$6 \quad$ Welche der folgenden Aussagen zur Endomyokardbiopsie ist richtig?

A Um den „sampling error“ möglichst niedrig zur halten, sollten möglichst viele EMBs entnommen werden.

B Der „sampling error“ ist bei der akuten Myokarditis niedriger als beim chronischen Verlauf.

C Die histologische Diagnostik der Myokarditis nach den Dallas-Kriterien ist der immunhistologischen Diagnostik überlegen.

D Eine EMB-Entnahme aus dem linken Ventrikel ist der einer rechtsventrikulären Entnahme hinsichtlich des Inflammationsnachweises überlegen.

E Eine EMB ist nur indiziert, wenn die vorausgegangene selektive Koronarangiografie keine Auffälligkeiten zeigt.

7 Für welche der folgenden Viren ist die Untersuchung peripherer Blutzellen zum Nachweis einer kardialen Virusinfektion möglich?
A Adenovirus
B Enterovirus
C chromosomal integriertes HHV 6
D Parvovirus $B_{19}$
E Zytomegalievirus

8 Für welche der folgenden Fragestellungen ist die Untersuchung der RNA aus Endomyokardbiopsien wichtig?
A Nachweis von Borrelien
B Enterovirusinfektion
C Adenovirusnachweis
D Influenzaviren
E Hepatitis-B-Virus

9 Welche der folgenden kardiotropen Viren können durch eine Interferon- $\beta$-Behandlung effektiv eliminiert werden?
A Epstein-Barr-Virus
B Enterovirus
C Parvovirus $\mathrm{B}_{19}$
D Zytomegalievirus
E Hepatitis-C-Virus

10 Bei welchen der folgenden Krankheitsbilder ist eine immunsuppressive Therapie nicht indiziert?
A Virusmyokarditis
B Riesenzellmyokarditis
C virusnegative, inflammatorische Kardiomyopathie
D Sarkoidose
E eosinophile Myokarditis 